Objectives: To deepen the knowledge about suicide prevention apps and understand its usefulness.

Materials and Methods: Non-systematic review using Pubmed with the keywords: " suicide prevention app" and "suicide prevention mobile app".

\title{
Suicide and technology
}

Suicide is a major public health issue and has a large impact on the lives of many people. Death by suicide is a complex situation and is usually preceded by suicidal thoughts, plans, and attempts. Dramatic advances in data science and wearable technology may provide new avenues to recognize suicidal behavior, prevent suicide, and save lives. 4

\section{Reasons for a mobile app}

The reasons for developing a mobile application are numerous. Smartphones are omnipresent, people have them at all times and in all places. Apps can help users to detect distress and offer fast contact to a support hotline. 1

\author{
Features mostly used in suicide prevention apps: \\ * Information, education and training \\ * Resource locators \\ * "Emergency button" features \\ * Safety planning and other coping tools \\ * Clinical assessment and automated intervention. 1
}

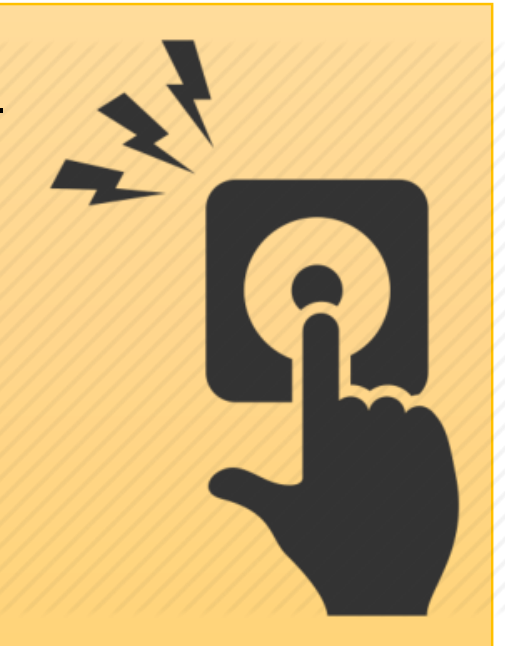

\section{USES OF SUICIDE PREVENTION MOBILE APPS:}

* In addition to pharmacological and psychotherapeutic treatment,

* People who are reluctant or unable to find professional treatment. 1
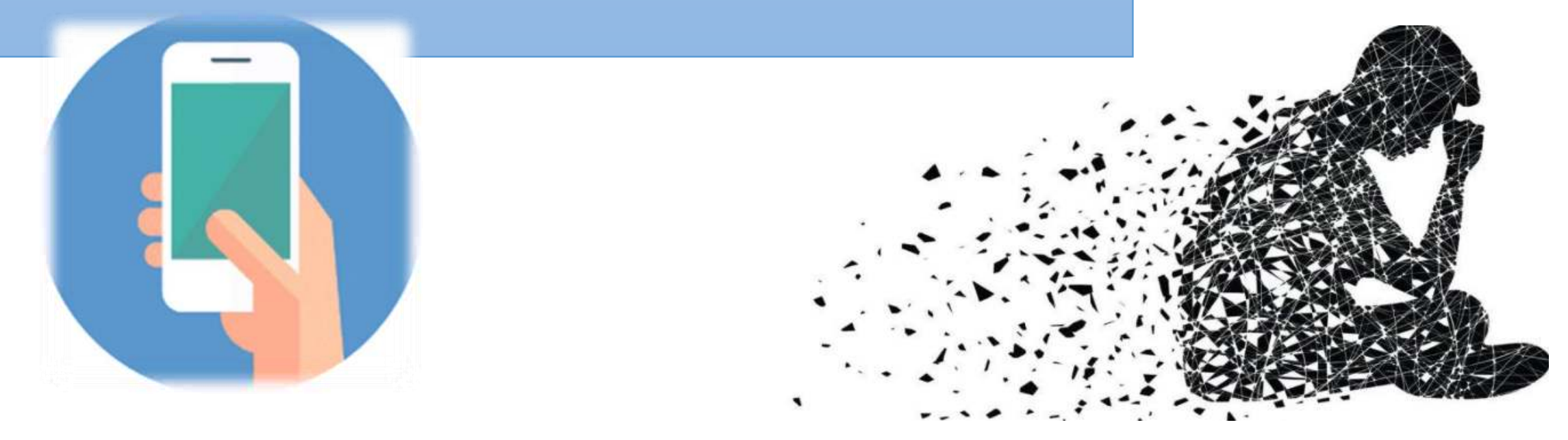

Although severe suicidal ideation
requires professional treatment, many
suicidal persons do not overcome
help-seeking barriers such as not
perceiving a need for help, access to
and cost of services, fear, and stigma. 2

ONLINE TOOLS AND PROGRAMS FOR SUICIDE PREVENTION CREATE NEW POSSIBILITIES DUE TO THEIR:
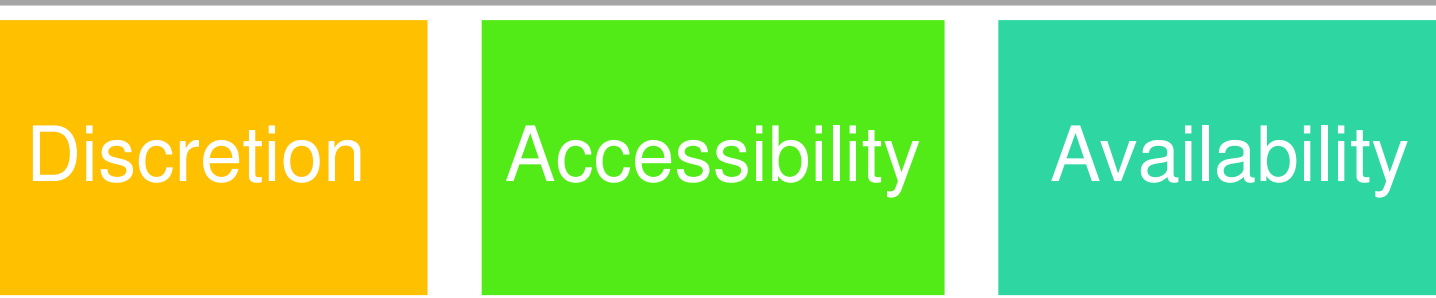

which are important barriers for help seeking. 1

\section{WORRYING FACTS}

the vast majority of apps were little more than checklists of symptoms or resources and also some apps contained information that could be potentially harmful or may worsen a person's condition. 4

\section{Developing an app}

In this study called Ibobbly mobile health intervention for suicide prevention in Australian Indigenous youth: a pilot randomised controlled trial, the authors created an app for suicide prevention and used it in Australia. A two-arm randomized controlled trial (one intervention group and a 6-week waitlist control group) was conducted, with post-test, and 6-week-followup.

This study found that an app offering acceptance-based therapy reduced depression symptoms and psychological distress in the target population, but did not lead to significant changes on suicidality or impulsivity.

They examined whether change in ideation was present for those with ideation $(n=45)$, but there were no differences found in this subanalysis. 3
Low cost

\section{What does the future hold?}

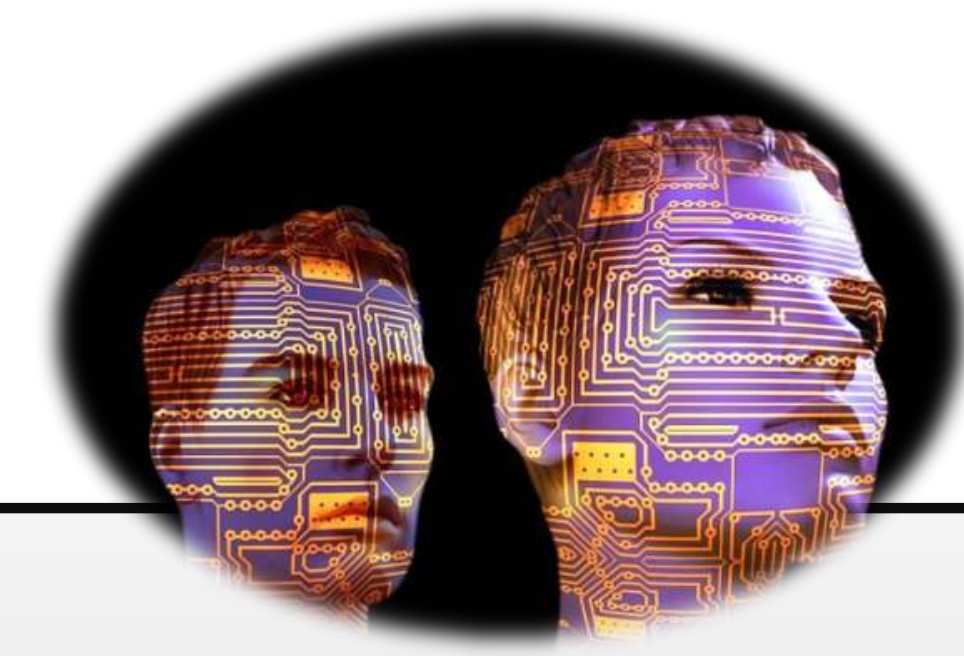

Detect suicidal thinking through speech and facial emotions. People who become suicidal may have differences in the sound frequency of their speech, either going from high frequency to low frequency or vice-versa. This could be a rapid way to assess suicidality, especially in high risk individuals who may not verbally disclose the presence of suicidal thoughts or plans.4

\section{Take Home Message:}

Despite the scarce evidence on the effectiveness of mobile applications for suicide prevention, smartphone applications can offer new opportunities, especially when it comes to providing suicide prevention and crisis intervention tools that can be used when in crisis.

\section{REFERENCES}

1. Aerts, Saskia, and Kirsten Pauwels. "BackUp: Development and Evaluation of a Smart-Phone Application for Coping with Suicidal Crises." PloS One, June 2017

2. Larsen, Mark Erik, et al. "A Systematic Assessment of Smartphone Tools for Suicide Prevention." PLOS ONE, Public Library of Science, Apr. 2016

3. Tighe, Joseph, et al. "Ibobbly Mobile Health Intervention for Suicide Prevention in Australian Indigenous Youth: a Pilot Randomised Controlled Trial." BMJ Open, British Medical Journal Publishing Group, 1 Jan. 2017

4. Vahabzadeh, Arshya, et al. “Digital Suicide Prevention: Can Technology Become a Game-Changer?” Innovations in Clinical Neuroscience, Matrix Medical Communications, 1 June 2016 